\title{
Control Design for System with Lipschitz Nonlinearity of State Derivative Variables in Reciprocal State Space Form
}

\author{
Yuan-Wei Tseng ${ }^{1}$ \\ ${ }^{1}$ Department of Electrical Engineering, I-Shou University, Kaohsiung 84001, Taiwan, R.O.C.
}

\begin{abstract}
This paper addresses the need of the novel reciprocal state space (RSS) form to be the supplement of standard state space system in control designs. Controller is designed utilizing state derivative feedback alone in RSS form for systems with Lipschitz nonlinearity. It will show that the design procedure is straightforward. It will also show via a real electric circuit that control for a class of singular systems with impulse modes can easily be carried out using the proposed design method. The purpose of this paper is to develop novel and simple method based on state derivative feedback so that wider ranges of problems can be solved without too much of mathematics overhead.
\end{abstract}

Keywords: reciprocal state space (RSS) form, state derivative feedback, Lipschitz nonlinearity, singular system, nonlinear control

\section{Introduction}

In many practical applications such as flight controls and especially robotic controls which contain nonlinear triangular function terms, there are nonlinear Lipschitz terms [1-2] as shown in the following state space system.

$$
\begin{gathered}
\dot{x}=\bar{A} x+\bar{B} u+\varphi(x, u) \\
\|\varphi(x, u)\|=\|\dot{x}-\bar{A} x-\bar{B} u\| \leq \rho\|x\|
\end{gathered}
$$

where $x$ is state vector, $u$ is control input vector, $\varphi(x, u)$ is a nonlinear Lipschitz term containing mismatched uncertainty and un-modelled dynamics and $\rho$ is the Lipschitz gain.

Generally speaking, for the system with Lipschitz nonlinear term, the changing rate of trajectory is limited by the changing rate of state. It is a highly active research area in academic. Methods based on Lyapunov stability [3-4], $H_{\infty}$ control [5][7] and LMI [3-4][6] have been successfully carried out to handle control designs for system with Lipschitz nonlinearity. In previous studies, only Lipschitz nonlinearity of state was considered. The studies of Lipschitz nonlinearity of state derivative were few if any. The majority of design methods was developed in state space form and applied state related feedback in control design. However, in many applications, the sensors directly measure state derivatives rather than states. For instance, accelerometers [8] in micro and nanoelectromechanical systems and structural applications are such cases because acceleration signals can only be modelled as state derivatives [9-10]. Consequently, abundant control algorithms with state related feedback developed in standard state space form cannot be readily applicable in this situation. Additional integrators which may increase the cost and complexity of the implementation are needed. Furthermore, for system in the following generalized state space form,

$$
E \dot{x}=F x+N u
$$

If $E$ in (2) is singular, the system has poles at infinity and is called singular system. It is obvious that singular system cannot be expressed in state space form to carry out control design. To provide supplementary design algorithms of state derivative feedback in state space form and handle some of singular systems, a direct state derivative feedback control scheme was developed in "Reciprocal state space" (RSS) form [9][11-15] by the author of this paper. If $F$ in (2) is invertible, we can have the following system in RSS form and carry out control design with state derivative feedback.

$$
\begin{gathered}
x=F^{-1} E \dot{x}-F^{-1} N u=A \dot{x}+B u \\
u=-K \dot{x}
\end{gathered}
$$


For above reciprocal state space (RSS) systems, state vector can be explicitly expressed in terms of state derivative vector and control input vector. The controllability and observability analyses for system in RSS form have been investigated in [12-13]. It shows that they turn out to be the same as their counterparts in state space form. After apply full state derivative feedback control law in ( $3 b)$, the closed loop system becomes

$$
x=(A-B K) \dot{x}=A_{c} \dot{x}
$$

The concept of RSS is based on a fact that for a non-singular matrix, the eigenvalues of its inverse matrix must be the reciprocals of its eigenvalues. Therefore, the eigenvalues of $A_{c}$ in (4) are the reciprocals of the closed loop system poles. To address this nature, the name of reciprocal state space form was given. If state derivative feedback gain $K$ can be designed such that the real parts of all eigenvalues of $A_{c}$ in (4) are strictly negative, the closed loop system in RSS form in (4) can achieve globally asymptotically stable. When a controllable system has no open loop pole at zero, it can be expressed in RSS form to carry out state derive related feedback control designs.

In this paper, the algorithm of utilizing state derivative feedback in RSS form to control system with Lipschitz nonlinearity of state derivative is introduced. To author's best knowledge, no related research has been reported. The main purpose of this paper is to take the advantage of RSS so that state derivate feedback can be systematically applied in dealing with nonlinear Lipschitz term to handle wider range of control problems.

\section{Control Design For System With Lipschitz Nonlinearity Of State Derivative Variables}

Consider the following controllable system in RSS form with Lipschitz nonlinearity function of state derivative.

$$
\begin{aligned}
& x=A \dot{x}+B u+\Phi(\dot{x}, u) \\
& \|\Phi(\dot{x}, u)\|=\|x-A \dot{x}-B u\| \leq \gamma\|\dot{x}\|
\end{aligned}
$$

where $\gamma$ is Lipschitz gain.

Without loss of generality, it is assumed that the matrix $A$ is Hurwitz. If the matrix $A$ is not Hurwitz, since $(A$; $B$ ) is controllable, a preliminary control can be used to make it Hurwitz. Therefore, we first focus on the controller design to eliminate the effect of Lipschitz nonlinearity to guarantee closed loop stability.

The following state derivative feedback control law is proposed.

$$
u=-\frac{K}{\|B\|^{2}} \dot{x}
$$

Substituting (6) to (5a), the closed loop system is

$$
x=\left(A-\frac{B K}{\|B\|^{2}}\right) \dot{x}+\Phi(\dot{x}, u)=A_{c} \dot{x}+\Phi(\dot{x}, u)
$$

To determine $K$, the following Lyapunov function candidate is selected

$$
V(x)=x^{T} P x
$$

where $P$ is a symmetric positive definite matrix. Applying (7), the time derivative of the Lyapunov function candidate becomes

Since

$$
\begin{aligned}
& \dot{V}(x)=\dot{x}^{T} P x+x^{T} P \dot{x}=\dot{x}^{T} P\left[A_{c} \dot{x}+\Phi(\dot{x}, u)\right]+\left[A_{c} \dot{x}+\Phi(\dot{x}, u)\right]^{T} P \dot{x} \\
& =\dot{x}^{T}\left(P A_{c}+A_{c}^{T} P\right) \dot{x}+2 \dot{x}^{T} P \Phi(\dot{x}, u) \\
& \because\|\Phi(\dot{x}, u)\| \leq \gamma\|\dot{x}\| \Rightarrow 2 \dot{x}^{T} P \Phi(\dot{x}, u) \leq 2 \gamma\|P \dot{x}\| \cdot\|\dot{x}\| \\
& \therefore \dot{V}(x)=\dot{x}^{T}\left(P A_{c}+A_{c}^{T} P\right) \dot{x}+2 \dot{x}^{T} P \Phi(\dot{x}, u) \leq \dot{x}^{T}\left(P A_{c}+A_{c}^{T} P\right) \dot{x}+2 \gamma\|P \dot{x}\| \cdot\|\dot{x}\|
\end{aligned}
$$

$$
\dot{x}^{T}(\gamma I-P)^{T}(\gamma I-P) \dot{x}=\dot{x}^{T} P P \dot{x}-2 \gamma \dot{x}^{T} P \dot{x}+\gamma^{2} \dot{x}^{T} \dot{x}=\dot{x}^{T} P P \dot{x}-2 \gamma\|P \dot{x}\| \cdot\|\dot{x}\|+\gamma^{2} \dot{x}^{T} \dot{x} \geq 0
$$


Using (12), the following inequality is obtained.

$$
2 \gamma\|P \dot{x}\| \cdot\|\dot{x}\| \leq \dot{x}^{T} P P \dot{x}+\gamma^{2} \dot{x}^{T} \dot{x}
$$

With (11) and (13), one can further obtain the following inequality

$$
\dot{V}(x) \leq \dot{x}^{T}\left(P A_{c}+A_{c}^{T} P\right) \dot{x}+\dot{x}^{T} P P \dot{x}+\gamma^{2} \dot{x}^{T} \dot{x}=\dot{x}^{T}\left(P A_{c}+A_{c}^{T} P+P P+\gamma^{2} I\right) \dot{x}
$$

Assuming that $\eta>0$ and

$$
\dot{x}^{T}\left(P A_{c}+A_{c}^{T} P+P P+\gamma^{2} I\right) \dot{x}=-\eta \dot{x}^{T} \dot{x}
$$

One can have

$$
\dot{V}(x) \leq-\eta \dot{x}^{T} \dot{x}<0
$$

Therefore, the closed loop system is asymptotically stable. Applying (6), (16) becomes

$$
P A_{c}+A_{c}^{T} P+P P+\left(\gamma^{2}+\eta\right) I=P A+A^{T} P-P \frac{B K}{\|B\|^{2}}-\frac{K^{T} B^{T}}{\|B\|^{2}} P+P P+\left(\gamma^{2}+\eta\right) I=0
$$

Let

$$
K=\frac{1}{2} B^{T} P
$$

, (17) becomes the following Algebraic Ricatti Equation:

$$
P A+A^{T} P-P \frac{B K}{\|B\|^{2}}-\frac{K^{T} B^{T}}{\|B\|^{2}} P+P P+\left(\gamma^{2}+\eta\right) I=P A+A^{T} P+P\left(I-\frac{B B^{T}}{\|B\|^{2}}\right) P+\left(\gamma^{2}+\eta\right) I=0
$$

Since $\left(I-\frac{B B^{T}}{\|B\|^{2}}\right)$ is symmetric positive semi-definite and $\left(\gamma^{2}+\eta\right) I$ is symmetric positive definite, when $A$ is Hurwitz, there exists a symmetric and positive definite matrix $P$ as the solution of (19) if the associated Hamiltonian matrix in (20) is hyperbolic, namely, $H$ has no eigenvalues on the imaginary axis [3][16].

$$
H=\left[\begin{array}{cc}
A & I-\frac{B B^{T}}{\|B\|^{2}} \\
-\left(\gamma^{2}+\eta_{c}\right) I & -A^{T}
\end{array}\right]
$$

It is further proven in [3] that if $\sigma_{\min }(M)$ is the minimal singular value of matrix $M$ and the number $\delta(M, N)$ is defined as

$$
\delta(M, N)=\min _{\omega \in R} \sigma_{\min }\left(\begin{array}{c}
i \omega I-M \\
N
\end{array}\right)
$$

, the condition for $H$ being hyperbolic given by (20) can be simplified as follows.

$$
\gamma<\delta\left(A^{T}, \frac{\gamma}{\|B\|} B^{T}\right)
$$

If the matrix $A$ in (5) is not Hurwitz, since $(A ; B)$ is controllable, a preliminary control $u_{1}=-K_{1} \dot{x}$ can be used to make it Hurwitz.

$$
x=\left(A-B K_{1}\right) \dot{x}+B\left(u+K_{1} \dot{x}\right)+\Phi(\dot{x}, u)
$$

To increase design flexibility, an invertible matrix $T_{c}$ is used to perform similarity transformation.

$$
x=T_{c} x^{\prime} \Leftrightarrow x^{\prime}=T_{c}^{-1} x
$$

Applying (24), (23) is

$$
x^{\prime}=T_{c}^{-1}\left(A-B K_{1}\right) T_{c} \dot{x}^{\prime}+T_{c}^{-1} B\left(u+K_{1} T_{c} \dot{x}^{\prime}\right)+T_{c}^{-1} \Phi\left(T_{c} \dot{x}^{\prime}, u\right)=A^{\prime} \dot{x}^{\prime}+B^{\prime}\left(u+K_{1} T_{c} \dot{x}^{\prime}\right)+T_{c}^{-1} \Phi\left(T_{c} \dot{x}^{\prime}, u\right)
$$


According to the Lipschitz nonlinearity after coordinate transformation, one can have new Lipschitz gain $\gamma^{\prime}$ in the following inequality.

$$
\left\|T_{c}^{-1} \Phi\left(T_{c} \dot{x}^{\prime}, u\right)\right\| \leq \gamma^{\prime}\left\|\dot{x}^{\prime}\right\|
$$

If we apply the following control law:

$$
u=-K_{1} T_{c} \dot{x}^{\prime}-\frac{1}{\left\|B^{\prime}\right\|^{2}} K^{\prime} \dot{x}^{\prime}
$$

Substituting (27) to (25), one obtain

$$
x^{\prime}=\left(A-\frac{B^{\prime} K^{\prime}}{\left\|B^{\prime}\right\|^{2}}\right) \dot{x}^{\prime}+T_{c}^{-1} \Phi\left(T_{c} \dot{x}^{\prime}, u\right)
$$

Using similar derivation in (7)-(19), one have

$$
P_{c} A^{\prime}+A^{\prime T} P_{c}+P_{c}\left(I-\frac{B^{\prime} B^{\prime T}}{\left\|B^{\prime}\right\|^{2}}\right) P_{c}+\left(\gamma^{\prime 2}+\eta_{c}\right) I=0
$$

Solving this Algebraic Ricatti Equation to obtain $P_{c}$, we have

$$
K^{\prime}=\frac{1}{2} B^{\prime T} P_{c}
$$

From (24), (27) and (30), the gain of control law $u=-K \dot{x}$ for the original system is found as follows.

$$
K=K_{1}+\frac{1}{\left\|T_{c}^{-1} B\right\|^{2}} K^{\prime} T_{c}^{-1}
$$

\section{Design Example For Singular System With Impulse Mode}

The following circuit shown in Figure 1 is an example of a singular system with impulse mode [17]. It is generally considered to be difficult to carry out the control design for such a system. However, we will find that, simply transforming the dynamic equations of the system into the RSS form in the first place, the control design becomes as straightforward as the usual control design approach used for standard state space systems.

Example: Consider the singular system of the circuit shown on the left-hand side of Fig. 1, and its equivalent alternating current circuit is shown on the right.

In the figure, $v_{C}$ is the capacitor voltage, $i_{E}$ is the emitter current, and $\alpha$ is the common-base current gain of the bipolar junction transistor. The dynamic equation for the system is given as

$$
\left[\begin{array}{ll}
C & 0 \\
0 & 0
\end{array}\right]\left[\begin{array}{l}
\dot{x}_{1} \\
\dot{x}_{2}
\end{array}\right]=\left[\begin{array}{ll}
0 & 1 \\
1 & 0
\end{array}\right]\left[\begin{array}{l}
x_{1} \\
x_{2}
\end{array}\right]+\left[\begin{array}{l}
0 \\
1
\end{array}\right] u,
$$

where $x=\left[\begin{array}{c}v_{c} \\ i_{E}\end{array}\right]=\left[\begin{array}{l}x_{1} \\ x_{2}\end{array}\right]$ and initial condition: $x(0)=\left[\begin{array}{c}1 \\ -0.5\end{array}\right]$.

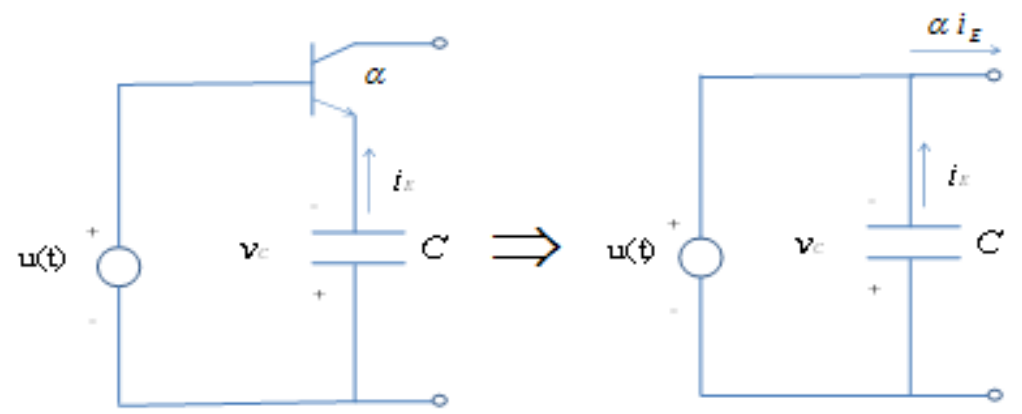


Fig. 1: Singular circuit system with impulse mode.

The system may be transformed into the RSS form:

$$
x=\left[\begin{array}{ll}
0 & 0 \\
C & 0
\end{array}\right] \dot{x}+\left[\begin{array}{c}
-1 \\
0
\end{array}\right] u
$$

For illustrational purpose, let $C=1$. If Lipschitz nonlinearity is added to the system as follows for verifying the proposed algorithm, we have

$$
x=\left[\begin{array}{ll}
0 & 0 \\
1 & 0
\end{array}\right] \dot{x}+\left[\begin{array}{c}
-1 \\
0
\end{array}\right] u+\left[\begin{array}{c}
0 \\
-2 \sin \dot{x}_{1}
\end{array}\right]=A \dot{x}+B u+\Phi(\dot{x}, u)
$$

Therefore, the minimum of Lipschitz gain is $\gamma=2$. Note that, greater Lipschitz gain can be selected in design if the performance of the closed loop system is not good enough. Since the open loop system is not Hurwitz, $u_{1}=-K_{1} \dot{x}=-\left[\begin{array}{ll}-1.5 & -0.5\end{array}\right] \dot{x}$, a preliminary control is used to make it Hurwitz. If $T_{c}$ is an identity matrix and $\eta=1$, the associated Hamiltonian matrix in (20) is obtained as follows.

$$
H=\left[\begin{array}{cccc}
-1.5 & -0.5 & 0 & 0 \\
1 & 0 & 0 & 1 \\
5 & 0 & 1.5 & -1 \\
0 & 5 & 0.5 & 0
\end{array}\right]
$$

Since the eigenvalues of $H$ are $-1.8297 \pm 0.4721 \mathrm{i}$ and $1.8297 \pm 0.4721 \mathrm{i}, H$ has no eigenvalues on the imaginary axis. Consequently, matrix $P_{c}$ in (29) should be symmetric and positive definite. By applying the proposed design process and solving Algebraic Ricatti Equation in (29), we do have a symmetric and positive definite matrix $P_{c}$ as follows.

$$
P_{c}=\left[\begin{array}{ll}
1.8534 & 0.3369 \\
0.3369 & 2.1594
\end{array}\right]
$$

Applying (30) and (31), the gain of control law for the original system is obtained as follows.

$$
K=\left[\begin{array}{ll}
-1.5 & -0.5
\end{array}\right]+\left[\begin{array}{ll}
-0.9267 & -0.1684
\end{array}\right]=\left[\begin{array}{ll}
-2.4267 & -0.6684
\end{array}\right]
$$

The closed loop poles which are the reciprocals of the eigenvalues of $A-B K$ are -11.1614 and -0.3850 .

The simulation of state responses of the close loop system is given in the following Fig. 2 and they do converge as expected. Therefore, the proposed methodology is successfully verified. 


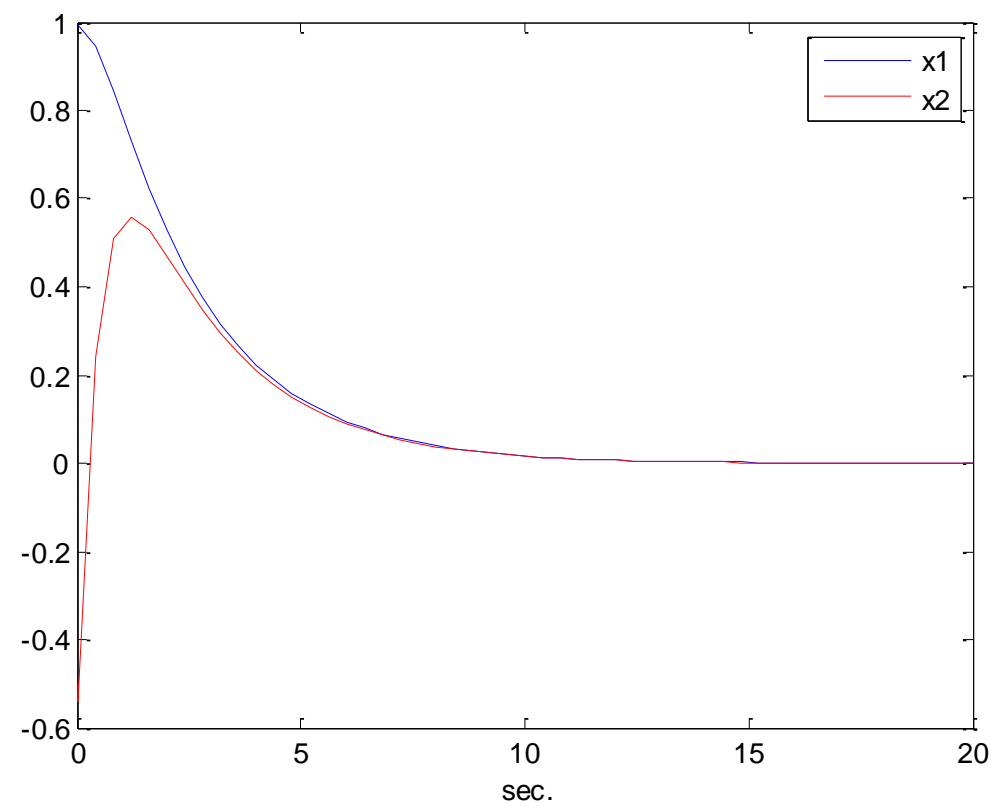

Fig. 2: state responses of the close loop system

\section{Conclusions}

Control design for system with Lipschitz nonlinearity of state derivative variable in RSS form has been investigated in this paper. It has shown via a real electric circuit that control design for a class of singular systems with impulse modes and Lipschitz nonlinearity can easily be carried out using the proposed method. The control design for system in RSS form using state derivative feedback can be obtained via an algebraic Riccati equation. Note that the derivation and design method parallel those for systems in standard state space form. Therefore, mathematics overhead is low for people to apply state derivative related feedback in RSS form.

\section{Acknowledgements}

The research reported here was supported by the Ministry of Science and Technology, Taiwan, under grants 103-2221-E-214 -045.

\section{References}

[1] S. H. Zak, "On the Stabilization and Observation of Nonlinear, Uncertain Dynamic Systems," IEEE Transactions on Automatic Control, 35(5), pp. 865-868, 1990.

http://dx.doi.org/10.1109/9.53535

[2] R. Rajesh, “Observer for Lipschitz Nonlinear Systems,” IEEE Transactions on Automatic Control, 43(3), pp.397-400, 1998.

http://dx.doi.org/10.1109/9.661604

[3] Y. L. Zhu, Adaptive output feedback control of nonlinear systems, Ph.D. Dissertation, Oklahoma State University, OK, USA, Dec., 2004.

[4] J. Song, S. P. He, "Finite-Time Passive Filtering for a Class of Uncertain Lipschitz Nonlinear Systems," Proceedings of the 32th Chinese Control Conference, pp. 369-374, July 26-28, 2013.

[5] Z. Li, X. Liu ,M. Fu ,L. Xie, "Global Hoo consensus of multi-agent systems with Lipschitz non-linear dynamics," IET Control Theory Appl., 6(13), pp. 2041-2048, 2012.

http://dx.doi.org/10.1049/iet-cta.2011.0555 
[6] Q. Fu, "Feedback Control for Nonlinear Systems with Quasi-one-sided Lipschitz Condition," Proceedings of the 29th Chinese Control Conference, pp. 309-311, July 29-31, 2010.

[7] A. M. Pertew, H. J. Marquez, and Q. Zhao, “Observer Design for Lipschitz Nonlinear Systems,” IEEE Transactions on Automatic Control, 51( 7), pp. 1211-1216 2006.

http://dx.doi.org/10.1109/TAC.2006.878784

[8] H. Kulah, J. Chae, N. Yazdi and K. Najafi, "Noise analysis and characterization of a S-D capacitive silicon microaccelerometers," IEEE Journal of Solid-State Circuits, 41, pp.352-361, 2006.

http://dx.doi.org/10.1109/JSSC.2005.863148

[9] Yuan-Wei Tseng, "Vibration control of piezoelectric smart plate using estimated state derivatives feedback in reciprocal state space framework," International Journal of Control Theory and Applications, vol. 2, no. 1, pp. 61-71, 2009.06.

[10] S. Fallah, A. Khajepour, B., Fidan, S. K. Chen, and B. Litkouhi, "Vehicle optimal torque vectoring using statederivative Feedback and linear matrix inequality,” IEEE Trans. Vehicular Technology, 62, pp.1540-1552, 2013. http://dx.doi.org/10.1109/TVT.2012.2232947

[11] Yuan-Wei Tseng, Control design of linear dynamic system with matrix differential equations for aerospace applications. Ph.D. Dissertation, Department of Aerospace Engineering, The Ohio State University, Columbus, Ohio, USA, 1997.

[12] Yuan-Wei Tseng, S. K. Kwak, and R. K.Yedavalli, "Stability, controllability and observability criteria for the reciprocal state space framework," Proceedings of the American Control Conference, pp. 5093-5097, 2003.

[13] Yuan-Wei Tseng, "Control designs of singular systems expressed in reciprocal state space framework with state derivative feedback," International Journal of Control Theory and Applications, 1; pp.55-67, 2008.

[14] Yuan-Wei Tseng, and Y. N. Wang, "Sliding Mode Control with State Derivative Output Feedback in Reciprocal State Space Form," Special issue of switched dynamics with its applications, Abstract and Applied Analysis, 2013;12 pages, Article ID 590524, 2013.

[15] Yuan-Wei Tseng, and Jer-Guang Hsieh, "Optimal control for a family of systems in novel state derivative space form with experiment in a double inverted pendulum system," Special issue of switched dynamics with its applications, Abstract and Applied Analysis. 2013; 8 pages, Article ID 715026, 2013.

[16] C. Aboky, G. Sallet, and J. C. Vivalda, Observers for Lipschitz non-linear systems, International Journal of Control, 75(3), pp.204-212, 2002. http://dx.doi.org/10.1080/00207170110107256

[17] R. W. Newcomb, "The Semistate description of nonlinear time variable circuits," IEEE Transactions on Circuits and Systems, vol. 28, no. 1, pp. 62-71, 1981.S. Chen, B. Mulgrew, and P. M. Grant, "A clustering technique for digital communications channel equalization using radial basis function networks," IEEE Trans. on Neural Networks, vol. 4, pp. 570-578, July 1993. 